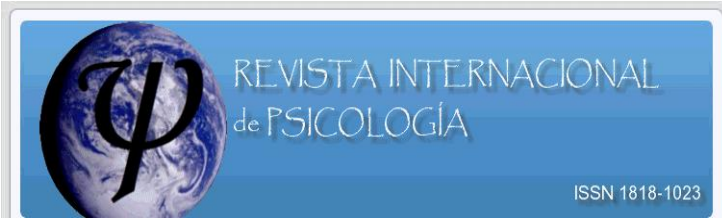

\title{
APLICACIONES DE LA TEORIA DEL APEGO EN EL TURISMO
} Lic. Maximiliano Korstanje ${ }^{1}$

Instituto de la Familia Guatemala

https://doi.org/10.33670/18181023.v8i01.44

\section{RESUMEN}

El siguiente trabajo se trata de una revisión de la teoría del apego y de la base segura dentro de la psicología evolutiva.

Los postulados de la teoría de la base segura apuntan a que el sistema de exploración se encuentra en estrecha relación con el sistema de conductas de apego y la figura de sus cuidadores. Las conductas que caracterizan y simbolizan la relación del adulto con su entorno se retrotraen a la edad temprana, en el momento en que el niño desarrolla su capacidad afectiva.

¿Qué diferencia existe entre alguien que decide volar a Inglaterra y aquel que se decide viajar a Mar del Plata? ¿Cómo se puede estudiar este tema de una manera sería y que indicadores son fiables para tal fin?

Estas tres preguntas fueron claves para comenzar la investigación. No obstante, el asunto comenzó a encontrar ciertas limitaciones vinculadas a la metodología que se debía utilizar. ¿Es posible utilizar sin ton ni son en el plano del ocio una teoría que aún demuestra ciertas inconsistencias en su aplicación clínica?

\section{PALABRAS CLAVES}

Teoría del apego - base segura - turismo

\begin{abstract}
The following work is intended to a revision on attachment theory.
\end{abstract}

The postulates of the theory of the sure base point that the system of exploration meets in narrow relation the system of conducts of attachment and the figure of the keepers. The conducts that characterize and symbolize the relation of the adult with the environment are carried back to the early age, in the moment in which the child develops the affective capacity

What difference does exist between someone who decides to journey to England and that one that one decides to travel to Mar del Plata? How it is possible to study this topic of on a manner trustworthy?

These three questions were key to begin the investigation. Nevertheless, the matter began to find certain limitations linked to the methodology that had to be in use. It is possible to use careless they on the leisure scope, a theory which still demonstrates certain inconsistencies in its own clinical application?

\section{KEY WORDS}

Attachment theory - sure base - tourism.

\footnotetext{
${ }^{1}$ Pontificia Universidad Católica Argentina. Correo electrónico: maxikorstanje@ hotmail.com
} 


\section{INTRODUCCIÓN}

"Nacer en el mundo significa ante todo, nacer de progenitores que no son exclusivos, ser criado por adultos que constituyen los elementos conductores de nuestros fragmentos de existencia”. (Natanson; en Shutz, 1974:17). Así se refería Maurice Natanson en el prólogo de El Problema de la Realidad Social, versión que finalmente el filósofo Alfred Schutz no llegó a revisar. El sentido de esta frase está asociado a la presencia de los padres en la crianza del niño, pero no en un momento exacto de la vida sino como continuación temporal, más precisamente sobre "su situación

biográficamente determinada",

En forma sumariada, puede afirmarse que de alguna $u$ otra manera, el individuo tiende a dirigir sus actos acorde a su sistema de socialización primario, aún en su vida adulta. De esta idea, se ha ocupado gran parte de la psicología y la psicología social, pero de todas las teorías disponibles existe una que es por demás atractiva: La teoría del apego y de la base segura de John Bowlby.

Los postulados de la teoría de la base segura apuntan a que el sistema de exploración se encuentra en estrecha relación con el sistema de conductas de apego y la figura de sus cuidadores. Las conductas que caracterizan y simbolizan la relación del adulto con su entorno se retrotraen a la edad temprana, en el momento en que el niño desarrolla su capacidad afectiva.

En efecto, cuando decidimos vacacionar o asistir a algún congreso debemos (indefectiblemente) ausentarnos del hogar por un lapso determinado de tiempo. ¿Aquellos que se han socializado dentro de un ambiente seguro y estable tienen más posibilidades de desplazarse o aventurarse a distancias más lejanas en comparación con aquellos que se han socializado bajo ambientes de presión y agresión?, compartiendo el mismo centro de origen ¿Qué diferencia existe entre alguien que decide volar a Inglaterra y aquel que se decide viajar a Mar del Plata? ¿Cómo se puede estudiar este tema de una manera sería y que indicadores son fiables para tal fin?

Estas tres preguntas fueron claves para comenzar la investigación. No obstante, el asunto comenzó a encontrar ciertas limitaciones vinculadas a la metodología que se debía utilizar. ¿Es posible utilizar sin ton ni son en el plano del ocio una teoría que aún demuestra ciertas inconsistencias en su aplicación clínica?

De cierta forma, tanto la teoría de la base segura como la del apego muestran ciertas cuestiones que deben ser resueltas y debatidas teleológicamente antes de aplicarlas en forma práctica.

Por ese motivo, este trabajo (en particular) tiene como objetivo sacar a la luz y someter (lo más objetivamente posible) los alcances y las limitaciones que han tenido estas teorías en el mundo de la psicología clínica. Si las apreciaciones de Bowlby son correctas, entonces esta tesis tendría alcances que hasta ahora no han sido demostrados. Por ejemplo, uno de los temas que hasta ahora continúa inexplorado por las ciencias sociales es la influencia de la base segura y las relaciones de apego con los progenitores en relación a la posibilidad de mantenerse alejado del hogar.

\footnotetext{
${ }^{2}$ Véase El problema de La Realidad Social de Alfred Schutz, 1974. Página 39.
} 
En un potencial abordaje y sólo de encontrarse que esta tesis es válida para estudiar el comportamiento de la vida adulta, se podrá inferir en la relación que existe entre la figura de los progenitores y los procesos de toma de decisiones a la hora de elegir un destino turístico.

\section{ESTADO DEL ARTE}

La primera cuestión que debe resolverse cuando se habla de la teoría de la base segura, es la definición misma de apego. Según Pla Vemengo se entiende por comportamiento de apego a "toda conducta por la cual un individuo mantiene o busca proximidad con otra persona considerada como más fuerte. Se caracteriza también por la tendencia a utilizar al cuidador principal como una base segura, desde la cual explorar los entornos desconocidos, y hacia la cual retornar como refugio en momentos de alarma".

He aquí dos elementos a los que el autor hace referencia en forma asociada pero que pueden ser analizados también por separado: la base segura y el apego. Tal y como han especificado los autores que estudian este tema, la figura de los padres en el niño parecería ser un elemento fundamental para definir el tipo de apego en la vida adulta.

Aunque se habían realizado intentos de estudiar el apego en patos y gansos ya por los inicios del 50; una de las primeras investigaciones que hacen referencia al tema son las que condujo Mary Ainsworth en laboratorios externos en Estados Unidos y África. Según el autor, cada vez que el niño se despegaba de la madre por un lapso de tiempo y luego se volvía a reunir con ella experimentaba una serie de comportamientos y sentimientos que fueron clasificados como seguro, ansioso evitativo y ansioso resistente. (Ainsworth, 1974;Vemengo, 2005;Bowlby, a1989; Casullo, 2004)

Estos hallazgos, van a ser tomados por Bowbly para la construcción de su teoría sobre la base segura. Según este autor, cuando el niño se separaba de su madre existían reacciones que se podían diferenciar por procesos mutuamente excluyentes pero integrados. A) Etapa de protesta, b) etapa de desesperación, c) etapa de desapego total y resignación. Para el autor, estas vivencias poseen una incidencia directa sobre la formación de la personalidad temprana y acompaña las conductas durante toda la vida adulta.

Mary Main, en 1985, se ocupó de realizar ciertos estudios donde se observaban ciertas correlaciones teóricas tomando como base las entrevistas con algunos padres y madres sobre sus experiencias propias y la relación con sus hijos. El autor encuentra tres formas de apegos combinadas a) seguro-autónomo, b) inseguro-desatendido, c) inseguropreocupado. (Main, 2001)

En trabajos posteriores, a través de una entrevista semi-estructurada, Main y Golwyn (1991) se propusieron recoger las supuestas "experiencias tempranas" de un grupo de individuos y el "significado" que le daba cada entrevistado al vínculo de apego en su etapa adulta (en Waters y Hamilton, 2000). Según sus hallazgos, los autores encontraron que:

a) Los individuos clasificados como de base segura eran capaces de narrar sus historias en forma integrada y coherente. 
b) Los clasificados como inseguros no eran capaces de articular su historia en forma coherente.

c) Los clasificados como indiferentes minimizaron el papel que ha tenido el apego y tienen dificultad para expresar sus sentimientos. Aunque (a su vez) idealizan ciertas experiencias.

d) Para aquellos que fueron asignados al grupo de los no resueltos, los relatos mostraban signos de abuso por parte del cuidador o de pérdida temprana.

Los trabajos de Main, dieron lugar para que otros investigadores siguieran desarrollando el tema.

En 1987, Hazan y Shaver encontraron que los sistemas de relaciones románticos en adultos encuentran ciertas pautas comunes en la relación con los padres en la primera socialización. El modelo de pareja, dicho de otra manea, está influido y condicionado por el modelo parental. (Hazan y Shaver 1987) (Casullo, 2004)

Se estimaba que a nivel mundial en 1985, el $65 \%$ de la población presentaba un tipo de apego seguro, mientras que el $20 \%$ era evitativo y el restante $14 \%$ ambivalente. Para 1995, la distribución había variado en 55\% seguro, $23 \%$ evitativo, $8 \%$ ambivalente y 15\% desorganizada (Lyons-Ruth, 1996) (Tapia Méndez y González Bravo, 2002).

Rosenstein y Horovitz (1996, en Jones, 1996) señalaron que los adolescentes con una tipología "indiferente" eran más proclives a manifestar trastornos disociales, como abuso de drogas, personalidad narcisista y paranoide, mientras que aquellos con un tipo de apego "preocupado" se orientaban hacia otras patologías tales como trastorno obsesivo compulsivo, histriónico, bordeline o esquizotípico (Méndez Tapia y González Bravo, 2002).

Es posible que el estado de humor y ciertos factores ambientales influyan en la interacción de la madre con el niño. Sobre esto se han ocupado algunos estudios como los de Isabella (1993) y Stevenson-Hinde y Shouldice (1995).

En 1998, Peter Fonagy descubrió la función reflexiva que surge de la relación de apego o mejor dicho de la internalización de ese vínculo, es intra-psíquica. La experiencia afectiva de la propia madre en conjunción con las demandas del niño y la simbolización que la madre atribuye a esos requerimientos. (Fonagy, 2004) (Vemengo, 2005).

El temperamento propio del niño, si bien, influye en la expresión en el momento de separación parece no alterar las clasificaciones que recibe por parte de los investigadores (Belsky y Rovine, 1987; Delgado 2004). Esto habla a las claras, de que existe dentro de la comunidad científica ciertas diferencias. El problema se da desde el momento en que se cuestiona que el apego sea (exclusivamente) causante de la personalidad.

Dicho en otras palabras, los rasgos del niño en conjunción con ciertas variables ambientales y propias del adulto conforman el tipo de vínculo entre ambos. Es probable que un niño que requiere (por problemas de salud) un constante cuidado desarrolle un tipo de apego inseguro, como también es factible que se forme por un trastorno compulsivo de alguno de sus padres. 
Por otro lado, no siempre existe una correlación fija y confiable entre el tipo de apego y la relación parental. Vaughan y otros (1985) no encontraron correlación entre el apego y el vínculo materno en niños que durante los dos años posteriores a su nacimiento experimentaron "day-care". Sin embargo, cuando el cuidado estaba a cargo de sus padres la correlación parecía ser más directa. Niños cuidados durante su infancia por terceros no parecen ajustarse el modelo de apego tradicional. Thompson (1986) ha señalado la posibilidad de que la "situación del extraño" no sea una herramienta fiable para medir el tipo de apego como se pensaba (Delgado, 2004:75)

En Argentina también se han realizado trabajos relacionados con el apego. Siguiendo a Hazan y Shaver, María Martina Maria Casullo (2004) ha enfocado el tema a través de la teoría de la base segura y su aplicación en el modelo que los adultos reproducen en los vínculos románticos. Lo novedoso de este enfoque radica en un elemento nuevo en el estudio de esta teoría: la capacidad de enamorarse. Por ese motivo, primero el autor debe ocuparse de resaltar el papel que ha tenido la cultura a lo largo de los años para darle su significado.

Por otro lado, debe revisar lo que ha dicho la bibliografía hasta el momento sobre las consecuencias del amor en el comportamiento del sujeto; de esa forma queda el camino despejado para la cuestión que fundamenta su trabajo: pasar de la teoría del apego en niños a la teoría del enamoramiento en adolescentes y adultos.

El método que utiliza Casullo para trabajar empíricamente con ambos conceptos consiste en una aplicación de la escala Bartholomew (1990). Esta escala fue aplicada en 800 adultos, el $50 \%$ de sexo masculino y el restante $50 \%$ femenino de entre 30 y 60 años de edad. Luego se sometieron los datos a un análisis factorial exploratorio de componentes principales, con criterio Kaiser y rotación varimax, calculando la fiabilidad por escala mediante un coeficiente de Cronbach.

Si bien, los resultados obtenidos validan la construcción teórica sobre los sistemas de apego en Ainsworth (1974), Main (2001), Bowlby (a1989) y Hazan y Shaver (1990) el problema principal que Casullo no puede resolver es la relación de una teoría basada empíricamente en datos elaborados en base a observaciones en niños y su relación en personas adultas.

Aunque lo menciona en su construcción teórica, una de las dificultades mayores que tiene la teoría de apego cuando se la quiere aplicar en adultos es que no considera lo que Alfred Shutz ha denominado "la situación biográfica determinada", es decir la propia historia del sujeto. Cae así, la teoría del apego también en un determinismo que años atrás le criticara tanto Ana Freud como Melanie Klein. (Vemengo, 2005)

El punto parece ser que los tipos de apego se manifiestan como funciones contextuales y no cabe ninguna duda que a medida que aumenta la interacción del individuo con su medio también se modifica su vínculo parental original. (Baker, 2002; Feeney y Cassidy, 2003)

Por otro lado, en la mayoría de los casos la metodología utilizada tanto por los precursores de la teoría del apego como por sus seguidores apunta a una entrevista o a la aplicación de cuestionarios semi estructurados que intentan revelar algún tipo de comportamiento presente para luego indagar "en lo que el entrevistado recuerda" de su 
relación de apego con sus padres, lo cual la hace - de alguna u otra manera- ciertamente polémica y poco fiable.

En cierta forma, "los daños y traumas psicológicos en una edad o faso específicas no tienen por qué dar por resultado tipos preditiblemente específicos de problemas clínicos ulteriores. Nada demuestra que así sea ... la mayor parte de los teóricos del apego, tal vez por basarse en la psicología académica, se demoraron en escoger la idea de Bolwby de que, si bien el apego es una perspectiva sobre la evolución, sobre las especies y sobre la díada individual, también constituye un modo de ver la experiencia subjetiva del infante en la forma de un modelo que hipotéticamente éste tiene de la madre”. (Stern, 2000:41-43).

Es pertinente aclarar que si bien muchos investigadores de todas partes del mundo han aplicado estas escalas en sus sociedades, los resultados fueron totalmente contrarios a aquellos que habían alcanzado tanto Ainsworth como Bowlby. Por ejemplo en Israel la mayoría arrojó un apego inseguro ambivalente; en Japón los resultados fueron similares con una evidente ausencia del apego evitativo. En Alemania, es precisamente el apego evitativo aquel que se distingue por sobre las otras tipologías. (Delgado, 2004:77).

El factor cultural parece refutar el carácter universal que se le ha dado a la teoría del apego en la bibliografía anglosajona clásica. Por este motivo es necesario (para llevar la investigación a buen puerto) ocuparse de un análisis crítico de esta teoría según su padre y fundador, John Bowlby.

\section{CRITICA A LA TEORÍA DE LA BASE SEGURA}

\section{EI Vínculo Afectivo entre niño v adulto}

El vínculo entre el niño y sus padres ha sido uno de los elementos centrales en la teoría del apego y la base segura. La mayor parte de las ideas que desarrolló Bowlby sobre este tema están en su obra Vínculos Afectivos que editara por primera vez en 1979 en inglés. En esa obra, el autor dedica un capítulo a la influencia que el psicoanálisis ha despertado en el estudio de la relación entre adulto y niño, como así también a la continuidad de la neurosis (derivada de esa relación temprana) en la adolescencia o la adultez.

Al igual que Freud, Bowlby presupone erróneamente que no respetar o cumplir las exigencias del infante aumenta la propensión al odio. Por otro lado, considera que se han encontrado datos comparativamente fiables que demuestran según sus palabras que "la experiencia relativa a un niño de corta edad que es separado de su madre nos proporciona un dramático ejemplo de este central problema de la sicopatología: la generación de un conflicto tan grande que fallan los medios normales para su regulación”. (Bowlby, b1986:25)

¿Qué evidencia y que metodología sigue Bowlby para hacer tal afirmación? ¿Qué pruebas aporta para poder confirmar que la relación parental influye -en la manera que él dice que lo hace- en las conductas adultas?

En uno de los experimentos de Haenicke (1956) se compararon respuestas de un grupo de niños de entre 15 y 30 meses; parte de ellos eran de una guardería residencial 
mientras que los otros de una guardería de día. ${ }^{3}$ Según los resultados reportados: que los niños de la guardería residencial mostraban una tendencia a la hostilidad mayor que los de la otra guardería.

Desde este punto de vista, Bowlby une estos resultados a su hipótesis central sobre la separación madre-hijo, no sin caer en el error de prejuzgar la situación. Si bien, el trabajo de Haenicke prueba que existen diferencias conductuales entre ambos grupos de niños, es difícil poder creer que la conducta hostil sea dirigida hacia los padres ausentes, y si lo es, que ésta perdure a través de los años -sin que el niño pueda reencauzarla hacia otra figura sustituta-.

El segundo problema que enfrenta el autor, es que considera por un lado que los castigos son generadores de ansiedad, resentimiento y odio, mientras por el otro atribuye (erróneamente) que en las civilizaciones occidentales es común creer que el castigo es eficaz como herramienta de control. La aplicación sistemática de procedimientos coercitivos crea personalidades temerosas y ansiosas. (Bowlby, b1986:30)

Aunque esto pueda parecer cierto en algún aspecto de nuestra vida individual, la represión (coacción) como sistema integrador no parece ser monopolio de las sociedades occidentales, como bien lo observara Margaret Mead en sus trabajos etnográficos -cuando analiza la sociedad Mundugumor- (Mead, 1994:160-180).

Sin embargo, el principal obstáculo del cual Bowlby no puede desprenderse es su constante juicio de valor sobre la conducta del infante, entre lo que "está bien y mal". En uno de sus capítulos señala "los correspondientes parecen surgir con mucha mayor frecuencia a causa de tener los propios padres dificultades emocionales de las cuales tan sólo, en parte poseen conciencia y que no pueden controlar. (Bowlby, b1986:32)

Esas "dificultades" son transmitidas "inconscientemente" de los padres a los hijos. Dicho de otra manera, en la vida adulta el sujeto tiende a repetir los comportamientos que absorbió en la temprana edad. Pero esto trae aparejado un error conceptual gravísimo, el cual implica que la seguridad del niño/adulto esta supeditada al comportamiento socialmente esperado, mientras que cualquier conducta diferente (patología) asume un trauma anterior.

Por ejemplo, España recibe diariamente miles de turistas de todas partes del globo quienes intentan conseguir una entrada para "Las corridas de Toros" o "espectáculos afines". ¿Quién determina en ese contexto que es una actitud hostil o agresiva?. Obviamente que la postura de la sociedad protectora de animales con respecto a los organizadores del evento será diferente (supuestamente), según sus normas formales e informales. Una patología (conducta hostil o no) no dependerá en tal caso de la socialización primaria per se, sino más bien otros aspectos tales como la cultura, la organización social, y sus procesos de control.

Llegado el caso, el concepto de trauma (psicoanalítico) poco tiene que ver con lo que socialmente se considera una desviación. Es decir, los comportamientos y las conductas individuales se someten a procesos sociales de control que los condicionan (como bien

\footnotetext{
${ }^{3}$ La diferencia entre una guardería residencial de una de día es el tiempo que los infantes permanecen alejados de sus padres.
} 
observó Freud en el desarrollo de su tesis de sublimación), sin embargo la posibilidad de un cambio que transforme esos procesos de control implicará un nuevo orden social que según sus nuevos preceptos considere "lo que antes era patológico" como normal; y viceversa. A esta reflexión Bowlby no parece dedicarle mucho esfuerzo.

En forma sumariada, y después de todo lo expuesto, se puede afirmar que la unidimensionalidad con la que el autor parece analizar el tema lo lleva a no tener en cuenta dos aspectos, (en el desarrollo teórico de su trabajo); a saber: a) la consideración temporal entre un antes y un después, y b) la omisión del cambio social como elemento condicionante de las estructuras normativas.

\section{Las influencias del Psicoanálisis v la etología.}

"Aquellos que experimentaron en su niñez una intensa ambivalencia hacia sus padres o hermanos y que luego recurrieron inconscientemente a algunos de los primitivos y precarios medios de resolver conflictos de los que he tratado ya anteriormente (represión, desplazamiento y proyección ... no están preparados para la renovación del conflicto cuando llegan a ser padres. En lugar de reconocer la auténtica naturaleza de sus sentimientos hacia el hijo y de adaptar su comportamiento de acuerdo con ello, se encuentran movidos por fuerzas que no conocen y perplejos por sentirse incapaces de ser tan cariñosos y pacientes como desearían”. (Bowlby, b1986:35)

De hecho, el autor (entonces) reconoce que de no manifestarse ciertos mecanismos defensivos, el padre estaría en condiciones de resolver y no repetir los actos del cual ha sido sometido en su niñez. La teoría del apego es heredera de dos teorías que conllevan la misma dificultad: el psicoanálisis de Sigmund Freud y la tesis evolutiva de Charles Darwin.

Por un lado, la teoría psicoanalítica si bien hace una "elocuente" descripción simbólica de los movimientos inconscientes no puede proveer material empírico para la refutación o validez de la misma. (Schuster, 2004:393) ${ }^{4}$. Asimismo por el otro, la etología cae involuntariamente en la suposición de que la vida social está dividida etapas que determinan el comportamiento de las diferentes especies y su adaptación ambiental. (Darwin, 1959) ${ }^{5}$

No obstante, Bowlby no considera la posibilidad (siquiera) de que no exista relación entre la socialización primaria y la conducta propia en la vida adulta. Y si así es entonces ¿cómo probar tal conclusión?, ¿qué experimentos o evidencia empírica demuestran que existe una relación conductual entre pasado y presente?, ¿la figura materna no es una construcción subjetiva?, ¿qué demuestra que esa percepción no es producto de la imaginación u mecanismos proyectivos en vez de un vínculo verdadero?

\footnotetext{
${ }^{4}$ En dicha compilación Alan Rush analiza bajo el título ¿es el psicoanálisis una pseudos ciencia? Los preceptos de Karl Popper sobre la falsabilidad que debe tener una ciencia. "Según Karl Popper, el psicoanálisis de Freud es una pseudo-ciencia debido a que no satisface los requerimientos lógicos y metodológicos de la falsabilidad empírica”. Fuente: Popper y Las Ciencias Sociales. Página 393. En Schuster F.

${ }^{5}$ Bowlby se pregunta a sí mismo ¿estamos seguros de que las conductas en aves y patos que supo observar Lorenz se condicen con la de los seres humanos? Para responderse a esta pregunta, el autor entabla un puente entre Freud y Lorenz utilizando el concepto de la prensa hidráulica. Sin embargo, esta noción es inverosímil. Si bien existen ciertas compatibilidades nada asegura que el comportamiento humano siga patrones basados en la teoría de la prensa hidráulica.
} 
Estas cuestiones quedan inconclusas en Vínculos Afectivos ${ }^{6}$; y el autor no presenta ninguna prueba consistente para responder esas preguntas mas que ciertos experimentos en animales y un cierto paralelismo en algunas conductas humanas. Si bien la relación con la madre lleva implícita toda una gama de posibilidades de estímulo respuesta, esto no resuelve de modo satisfactorio el asunto planteado anteriormente.

Según la experiencias de varios psicoanalistas compiladas por Root (1957) en pacientes con personalidad "bloqueada de afecto", existe una tendencia a haber experimentado un duelo que no fue procesado correctamente. De esta forma, el tratamiento apunta a volver a la primera fase del duelo ayudando al paciente a recuperar los sentimientos reprimidos de amor y odio (amor al evocar su presencia y odio como reprimenda por haberse ido). Pero esta relación debe estudiarse en el marco cronológico que corresponde.

En su trabajo la Base Segura editado en 1988 por vez primera, Bowlby, considera que su hallazgo puede compararse con los que han encontrado algunos exponentes de los círculos psicoanalíticos tales como: el falso si-mismo (Winnicott, a1960); la personalidad fronteriza (Kohut, 1971) o el síndrome esquizoide (Fairbairn, 1962).

Bowlby, señala que la importancia de los informes psicoanalíticos para profundizar y validar su teoría del apego. Pero su preocupación no parece enraizada en la misma teoría psicoanalítica, ya que el apego parece acercarse más al conductismo que al psicoanálisis. Según una interpretación personal, el interés del autor está dado por los resultados del psicoanálisis y no por psicoanálisis en sí. Por lo menos así parece, cuando toma los informes de tres pacientes que parecen experimentar un fuerte "sentimiento de dependencia hacia el analista" que Bolwby considera producto de la relación de apego en la infancia del sujeto.

Sin embargo, como él bien lo percibe "aun así, los críticos pueden perfectamente plantear dudas sobre la validez de lo que un paciente recuerda de su infancia y preguntar si la sucesión de acontecimientos recordados tenía sobre sus sentimientos el efecto que él implícitamente afirma". (Bowlby, a1989:69)

\section{La Teoría del Apego. sus alcances v limitaciones.}

"El comportamiento de apego es una forma de conducta instintiva que se desarrolla en el hombre, al igual que en otros mamíferos, durante la lactancia y tiene como finalidad o meta la proximidad de la figura materna. La función del comportamiento de apego consistiría en la protección contra depredadores. Tal conducta se muestra especialmente intensa durante la niñez, cuando está dirigida hacia figuras parentales, pero continúa activa durante la vida adulta, en la que generalmente es encauzada hacia alguna figura activa y dominante que, con frecuencia se trata de un pariente, pero también a veces de un jefe o alguna persona de más edad que pertenece a la comunidad" (Bowlby, b1986:111).

¿Cómo se activa el vínculo con la madre?

\footnotetext{
${ }^{6}$ El libro es una compilación de diversas conferencias y artículos que John Bowlby tuviera la oportunidad de publicar décadas atrás. La primer Conferencia se dio entre los meses de Abril y Mayo en 1956, la segunda en la primavera de 1957, la tercera en 1961, la cuarta en 1967, la quinta en 1968, la sexta en 1970 y la séptima en 1976. Es por ese motivo, los conceptos e ideas del autor deben ser comprendidas dentro de los contextos sociales en los cuales se desarrollaron las conferencias citadas.
} 
En su obra Una Base Segura el auto señala "la conducta de apego del niño es activada especialmente por el dolor, la fatiga y cualquier cosa atemorizante, y también por el hecho de que la madre sea o parezca inaccesible”. (Bowlby, a1989:15)

Según los experimentos de Klauss y Kennell (1975) el comportamiento de las madres después del parto (cuando tienen la libertad de hacer lo que desean) está inmediatamente orientada al contacto físico con el bebe. Ante este acto, el infante se muestra complacido estableciendo su primer lazo con la madre o el cuidador. Luego se llevarán a cabo las primeras interacciones entre ambos con motivo de afianzar la relación. Durante este período se da una sincronía con la cual tanto el bebe como la madre se vinculan vocal y gestualmente.

Aquellos niños que han respondido sensiblemente a los estímulos maternos tienen menos posibilidades de resistirse a los deseos de sus padres, así parecía observar Mary Ainsworth (Bowlby, a1989:21).

Según los hallazgos de Frommer y O Shea (1973) las mujeres que durante su embarazo han presentado antecedentes de separación con sus propios padres antes de los once años de edad, están más predispuestas a interactuar menos con su hijo que aquellas que han tenido una infancia estable. Eso a grandes rasgos coincide con el concepto freudiano de neurosis.

Sin ir más lejos, Rutter (1979) encontró que las personas criadas en hogares hostiles o "quebrantados" tienen mayor posibilidad de formar matrimonios y hogares con esas mismas características.

Otros estudios encuentran que aquellos individuos que en su niñez han recibido maltratos son producto de un parto anormal o de haber sido separados de su madre por más de cuarenta y ocho horas. Además los niños maltratados parecían enfermar con mayor frecuencia que sus hermanos no maltratados (Lynch, 1975). Los resultados volvieron a ser validados cinco años más tarde por otros dos investigadores, Cater y Easton (1980).

Bowlby estaba convencido, según todos los casos que observó y pudo recopilar en La Base Segura $^{7}$, que la relación entre niño y padres a una edad temprana influía en la vida del sujeto hasta su adultez. El apego no sólo condicionaba el tipo de personalidad sino que también fijaba la manera de relacionarse con un modelo de autoridad.

Aunque la teoría de Bolwby parezca muy ilustrativa, ¿que tiene que ver entonces la relación de una figura de autoridad con la elección de un destino turístico? O precisamente ¿Qué trabajos certifican que el apego se correlaciona con la capacidad de exploración?

Para responder satisfactoriamente estas cuestiones, es necesario introducir a la base segura como elemento de análisis; precisamente sobre ella Bowlby advierte que "la provisión por parte de ambos progenitores de una base segura a partir de la cual un niño o un adolescente puede hacer salidas al mundo exterior y a la cual puede regresar sabiendo con certeza que será bien recibido, alimentado física y emocionalmente, reconfortado si se siente afligido y tranquilizado si está asustado ... sólo cuando el oficial que comanda la fuerza

\footnotetext{
${ }^{7}$ Muchísimos más de los que se han podido mencionar en este trabajo por una cuestión espacial.
} 
expedicionaria se siente seguro de sí mismo, su base tiene la certeza de que él se atreve a seguir adelante y correr riesgos". (Bowlby, 1989:24)

Algunos estudios, como los de Schaffer y Crook (1979) validan la hipótesis del autor, al observar una cierta correlación entre el apego y las técnicas de los primeros pasos del bebe cuando se decide comenzar a caminar.

Bolwby (mismo) reconoce que la ansiedad que manifiesta el individuo ante la pérdida o el duelo no se deriva del dolor como creía el psicoanálisis tradicional, sino que es consecuencia de un aumento en el riesgo y el peligro que se percibe del entorno; " $\mathrm{el}$ hombre al igual que otros animales, responde con temor a determinadas situaciones, no porque éstas conlleven un alto grado de dolor y peligro, sino porque indican un aumento del riesgo" (Bowlby, a1989:44). Esa ansiedad termina despertando ira hasta que en algunos casos se vuelve disfuncional para la propia persona.

Si la apreciación del autor no es equivocada, entonces la percepción que el sujeto tiene de su medio ambiente es producto de su relación parental. Siendo así, la capacidad de desplazarse como de explorar el ambiente sin necesidad de recurrir constantemente a una base (hogar) tiene cierta y seria vinculación con la figura materna o paterna. Esto presupone, entonces que las distancia que el sujeto está dispuesto a recorrer desde su base sin experimentar sentimientos de ambivalencia, temor o incertidumbre se explican a través de la relación sentimental con una figura de referencia. Aquellos con personalidad "segura" podrán recorrer mayores distancias en comparación con "evitativos" o "ambivalentes"

¿Ha podido Bowlby proveer experimentos empíricos sobre esta hipótesis?, ¿Qué validez científica muestran los mismos?

Mary Ainsworth en su proyecto de Baltimore encontró similitudes entre el comportamiento de los niños y la forma de exploración que éstos mostraban hacia el observador. Del grupo de niños catalogados como seguros la exploración se observaba activa; en el grupo ansioso, por llamarlo de alguna manera, las conductas de exploración eran pasivas en presencia de la madre. Luego el autor, mediante el estudio de la personalidad de los progenitores llega a la conclusión de que existen patrones de conducta comunes entre madre e hijo, las cuales también (presupone) fueron parte de la crianza de la propia cuando era hija.

Estos resultados, como bien señala Bowlby presenta una limitación importante. "aunque el descubrimiento de Ainsworth de una correlación entre la sensibilidad de una madre ante su hijo y el modo en que el hijo se comporta con ella a los doce meses es estadísticamente muy significativo y ha sido confirmado por sucesivos estudios, siempre es posible argumentar que quien desempeña el papel más importante al determinar si la interacción se desarrolla felizmente o no, es el niño y no la madre." (Bowlby, a1989:63)

En 1972, Anderson demostró según un estudio que durante el segundo o tercer año de vida un niño raramente se aleja de su madre más de sesenta metros antes de retornar. Si la pierde de vista, olvida la exploración ya que su deseo es volver con ella. Si el niño es pequeño atraerá la atención "haciendo berrinches" pero si es un niño de más edad emprenderá una búsqueda hasta dar con ella nuevamente. En base a esta observación Bowlby intuye que "podemos entonces dar por sentado que la conducta que lo aleja de su madre hacia el vasto mundo- que se denomina correctamente conducta exploratoria- es 
incompatible con la conducta de apego y tiene menor prioridad. Así, sólo cuando la conducta de apego está relativamente inactiva, tiene lugar la exploración ... a medida que un individuo se hace mayor, su vida continúa estando organizada del mismo modo, aunque sus excursiones se vuelven continuamente más prolongadas en el tiempo y en el espacio ...Durante la adolescencia pueden durar semanas o meses, y es probable que se busquen nuevas figuras de apego ... todos nosotros, desde la cuna hasta la tumba, somos muy felices cuando la vida está organizada como una serie de excursiones, largas o cortas, desde la base segura proporcionada por nuestra figura de apego." (Bowlby, a1989:78)

En pocas palabras, por el modelo de la base segura el sujeto tiene tres tipos de comportamientos posibles a la hora de explorar el entorno. Aquellos con un apego seguro se atreven a explorar el mundo que los rodea y se reconocen en él; luego están los que demuestran un apego ansioso resistente; llegado el caso estos individuos son propensos a aferrarse y demuestra rasgos de ansiedad ante la posibilidad de exploración; finalmente, de los que poseen un apego elusivo o ansioso elusivo, algunos diagnosticados como narcisistas, se ven propensos a la autosuficiencia y a tendencias autoritarias. Su capacidad de exploración es pobre y su inseguridad muy alta.

Según experimentos realizados por Sroufe (1985) la estabilidad de las pautas de apego tiende a perpetuarse a través del tiempo. A medida que el infante crece esas pautas van conformando su percepción de sí, y comienzan a condicionar las relaciones con los demás. Por un proceso de "internalización”, el autor está convencido de que según vínculo de apego entre madre-hijo, se puede predecir el comportamiento del individuo tres años y medio más tarde. Estos descubrimientos si bien refuerzan las hipótesis de Mary Main y otros, no existen pruebas o trabajos que prologuen la observación por un lapso más largo. Esto trae consigo algunas complicaciones de tipo teóricas que se analizaran en el siguiente apartado.

\section{El problema de los dos tiempos. (Discusión final)}

De eso se trata lo que ha sido denominado "el problema de los dos tiempos". Generalmente, existen dos formas de abordar un problema en ciencias sociales. Una de ellas, es bajo una perspectiva sincrónica la cual presupone que los resultados son válidos únicamente para el tiempo en el que se aplicó la investigación. De hecho, los experimentos de Heinicke recurren a un estudio realizado en una esfera sincrónica; que los niños mostraran comportamientos diferenciales hacia sus madres dependiendo del contacto que tuvieran con ellas, es un aspecto que habla de esos niños en ese espacio y en ese tiempo. Dentro de esos resultados se excluyen por ejemplo a los adultos (que ya no son niños) de Rusia del año 1975.

En cambio, un trabajo diacrónico hace referencia al estudio de un objeto comprendido en dos o más tiempo. Las historias clínicas son el reflejo de este tipo de casos; entonces el analista o psiquiatra estudia en un lapso de tiempo a uno o varios individuos. Sin embargo, los experimentos que cita Bowlby si bien cumplen -en algunos casos- con la perspectiva sincrónica tienen muchos problemas para adaptarse a la diacrónica y si lo hace el lapso temporal es decididamente insuficiente.

Dicho en otras palabras, quizás esté probado que exista un apego especial (en determinada fase del crecimiento) entre el infante y su madre, tal vez esté probado que llegado el caso de cierta separación o pérdida existan comportamientos neuróticos en los niños; puede incluso mediante un estudio de años estudiar la evolución de ese niño hasta llegar a los 32 años. No obstante, al momento no existe un estudio de tal 
envergadura. Los casos que Bowlby recopila sobre adultos con problemas de relación llevan a presuponer que existe una distorsión en la percepción subjetiva o intra-psíquica del paciente pero que (quizás) nada tengan que ver con lo que realmente aconteció en su vida temprana. Por ese motivo, las escalas que tienden a medir este tipo de comportamientos no deben ser vinculadas a la niñez; de lo que poco se sabe mas que por declaraciones del paciente.

Ergo, de aplicar la teoría del apego a los comportamientos presentes en las vacaciones o en la toma de decisiones, una la postura sensata sería optar por una posición sincrónica que evalué la relación del sujeto con sus diferentes grupos de referencia y su influencia en los puntos a los cuales decida viajar. Por ejemplo, es mucho más coherente evaluar la relación que un individuo tiene con sus padres, con su trabajo y como influyen en la elección de su viaje en tiempo actual que intentar inferir la forma en que el apego temprano ha llevado a elegir Mar del plata en detrimento de Tokio (a la hora de emprender un viaje).

Afirma Bretherton (1985) lo importante en este tipo de cuestiones no es el apego o el vínculo que han tenido madre e hijo en el pasado sino su posterior interpretación y elaboración de esas experiencias, que es entre otras cosas lo que puede evaluar las herramientas y las escalas convencionales de apego (Delgado, 2004:70).

El tercer problema teórico que se suscita de la teoría de la base segura es que el objeto que brinda seguridad va cambiando a medida que pasa el tiempo. Tal como demostrara Winnicott con su teoría del objeto Transicional, la seguridad del sujeto no depende de una relación dada (aunque pueda estar influida por ésta) sino que puede ser variable en relación al tiempo y al objeto mismo, -llegado el caso el niño se desprende del amor maternal mediante un objeto que le sirve de transición para tal fin y que luego se desarrollará mediante el juego. (Winnicott, b1996:135)

Un sujeto con una personalidad ansiosa o evitativa puede sentirse inseguro en una relación sentimental pero demostrar la misma inseguridad en la elección de un viaje. La posibilidad que muestra el sujeto en volcar su ansiedad hacia un objeto implica indirectamente que se vea obligado a excluir a otros.

Por ese motivo, puede demostrar en una esfera de su vida privada cierta inseguridad como en la relación que tiene con su pareja pero experimentar seguridad en su ámbito laboral. Contrariamente, puede sentirse ansioso e inseguro en su vida profesional pero confiable en el afectivo. La posibilidad de que un sujeto manifieste un apego ansioso o evitativo en todas las esferas de su vida en la misma intensidad es, sin lugar a dudas, un fenómeno minoritario.

Finalmente desde un punto de vista emico, la tesis de la base segura muestra ciertas complicaciones para analizar y comprender las diferencias culturales como condicionante de los comportamientos tanto en lo individual como en lo grupal. Los resultados que han arrojado las investigaciones que se han realizado en diferentes partes del mundo han sido totalmente encontrados y poca similitud guarda con aquellas conducidas en los Estados Unidos de América.

Es posible que -de cierta manera-, tanto la teoría del apego como la base segura estén decididamente orientadas a caer en los mismos errores conceptuales en los que han 
caído sus predecesoras, pero esto es sólo una conjetura más. Por lo pronto, algunas consideraciones deben ser tenidas en cuenta a la hora de aplicar la teoría del apego y de la base segura en otros campos ajenos a la psicología clínica.

\section{REFERENCIA BIBLIOGRAFICA}

Ainsworth M. D

1974. The Development Of Infant-Mother Attachment. Review of Child

Development. Chicago Press, University of Chicago.

Anderson J.W

1972. "Attachment Behaviour out of Doors". Cambridge University Press, Cambridge.

Baker P. J

2002. "An Evaluation of Relational contextual variables that may influence stability across rating and attachment styles". Dissertation Abstract International, Section B63, 1-B.

Belsky J y Rovine M.

1987. "Temperament and Attachment: security in the Strange situation. An empirical reaprochment. Child Development, 58;787-792.

Bowlby J.

a) 1989. Una Base Segura: aplicaciones clínicas de la teoría del apego. Paidos, Buenos Aires.

b) 1986. Vínculos Afectivos: formación, desarrollo y pérdida. Editorial Morata, Madrid.

Bretherton I.

1985. "Atthachment Theory: retrospect and prospect. Child Development, 50:1-22.

Casullo A. M.

2004. "Vínculo de Apego Romántico en Adultos: escala de auto evaluación". Revista Psicodiagnosticar. (Publicación en curso)

Cater J. I y Easton P. M

1980. "Separation and Other Stress in Child Abuse". Lancet 1:972

Darwin C.

1959. The Origino of Species by Means of Natural Selection. Editorial Murray, Londres.

Delgado Oliva A.

2004. "Estado Actual de La Teoría del Apego". Revista de Psiquiatría y Psicología del Niño y del Adolescente, 4:55-69.

Fairbairn W.R.D.

1962. Estudio Psicoanalítico de la Personalidad. Editorial Hormé, Buenos Aires. 
Feeney B.C y Cassidy R.

2003. "Reconstructive Memory related to adolescent-parent conflict interaction: the influence of attachment related representations of immediate perceptions and changes in perception over time. Journal of Personality and Social Psycology, 85: 945-950.

Fonagy $\mathrm{P}$.

2004. Teoría del Apego y Psicoanálisis. Editorial Spaxs, Barcelona.

Hazan C. y Shaver P.

1990. "Love and Work: an attachment theoritical perspectiva". Journal of Personality and Social Phsycology, 59:270-275.

Heinicke C.

1956. "Some Effects of Separating Two-years Old Children from their Parents: a comparative study.” Revista Human Relations, 9:105-176.

Isabella R.A.

1993. "Origins of Atthachment: Maternal interactive Behavior across the first year. Child Development, 64:605-621.

Jones E.

1996. "Introduction to the Special section on Attachment and Psychopatology: part 1." Journal of Consulting and Clinical Psychology. Vol 64 (1):5-8.

Klaus M. H y Kennell J. H

1975. Maternal Infant Bonding. Editorial Mosby, Saint Louis.

Kohut H.

1971. The Analisys of the Self. International University Press, New York.

Main M.

2001. "Las Categorías Organizadas del Apego en el infante, en el niño y en el adulto: atención flexible versus inflexible bajo estrés relacionado con el apego". Revista de Psicoanálisis, Apertura Psicoanalítica. Número 8.

Mead M.

1994. Sexo y Temperamento. Editorial Altaya, Barcelona.

Lynch M.

1975. "Ill-health and Child Abuse", The Lancet.

Lyons-Ruth K.

1996. Attachment Relationships among children with agressive behaviour problems: the role of disorganized erly attachment patterns. Journal of Consulting and clinical Psychology. 64 (1):64-73. 
Schaffer H. R y Crook C. K. 1979. "The Role of the Mother in early Social Development", en B. McGurk (comp): Issues in childhood social development, 55-78, Londres.

Schutz A.

1974. El Problema de la Realidad Social. Amorrortu Editores, Buenos Aires.

Schuster F. (Compilador)

2004. Popper y Las Ciencias Sociales. Editores de América Latina, Buenos Aires.

Stevenson-Hinde J y Shouldice A.

1995. Maternal Interactions and self-reports related to attachment classifications at 4.5 years. Child Development, 65:583-590.

Tapia Méndez L. y González Bravo L.

2002. "Descripción de Patrones de Apego en Menores Institucionalizados con Problemas Conductuales". Revista de Psicología. 11 (2):75:92.

Thompson P.A

1986. "The Effects of Infant day care through the prism of attachment theory: a critical appraisal”. Early Childhood Research Quaterly, 3:273-280.

\section{Rutter M.}

1979. "Maternal Deprivation, 1972-1978: new findings, new concepts, new approaches". Child Development, 50:283-305.

Sroufe L.A.

1985. "Attachment clasification from the perspective of infant-caregiver relationships and infant temperament. Child Development, 56:1-14.

\section{Stern D.}

2000. El Mundo Interpersonal del Infante: una perspectiva desde el psicoanálisis y la psicología Evolutiva. Editorial Paidos, Buenos Aires.

Vaughan B.E y otros.

1985. "The Impact of Day-Care on Child Mother Attachment Quality: another look at some enduring questions. Child Development, 50:209.

Vemengo P.

2005. "Apego". Revista Psicoanálisis hoy, 4:1-4

Waters E. y Hamilton E.

2000. The Stability of Atthacment Security from Infancy to Adolescence en Early Adulthood: General Introduction. Child Development. 71 (3): 678-679

Winnicott D. W.

a) "Ego Distortion in Terms of Ture and False Self". The Maturational Facilitating enviromentm 140-52, International University Press, New York.

b) 1996. Realidad y Juego. Editorial Gedisa, Barcelona. 


\section{(a) (1)}

Este texto está protegido por una licencia Creative Commons

$$
\underline{4.0} \text {. }
$$

Usted es libre para Compartir — copiar y redistribuir el material en cualquier medio o formato - y Adaptar el documen- to - remezclar, transformar y crear a partir del material- para cualquier propósito, incluso comercialmente, siempre que cumpla la condición de:

Atribución: Usted debe reconocer el crédito de una obra de manera adecuada, proporcionar un enlace a la licencia, e in- dicar si se han realizado cambios. Puede hacerlo en cualquier forma razonable, pero no de forma tal que sugiera que tie- ne el apoyo del licenciante o lo recibe por el uso que hace.

$\underline{\text { Resumendelicencia - Textocompletodelalicencia }}$ 